\title{
A Virtual Reality Surgical Training System for Office Hysteroscopy with Haptic Feedback: A Feasibility Study
}

\author{
Vladimir Poliakov ${ }^{1,2} 凹$, Kenan Niu ${ }^{1}$, Bart Paul De Vree ${ }^{3,4}$, Dzmitry \\ Tsetserukou $^{2}$, and Emmanuel Vander Poorten ${ }^{1}$ \\ 1 Robot-Assisted Surgery Group, the Mechanical department, KU Leuven, Belgium \\ vladimir.poliakov@kuleuven. be \\ 2 Space Center, Skolkovo Institute of Science and Technology, Russia \\ 3 Departement of Obstetrics and Gynaecology, Ziekenhuis Netwerk Antwerpen, \\ Campus Middelheim, Belgium \\ 4 Departement of Obstetrics and Gynaecology, Universitair Ziekenhuis Antwerpen, \\ Belgium
}

\begin{abstract}
Hysteroscopy is a widely used gynaecological procedure to evaluate and treat cervical and intra-uterine pathology. In the last few decades, technical refinements in the optics technology, surgical accessories and the reduction of the outer diameter of the instrument have made it possible to perform many hysteroscopic procedures, including some operative procedures, in the office setting and without any anesthesia. Mini-hysteroscopic procedures in the office setting are associated with less pain, lower complication rate and faster recovery compared hysteroscopic procedures in day surgery under general anesthesia.

The main challenge for the clinician in performing office hysteroscopy is to pass the narrow cervical canal. Inaccurate motion or excessively applied force can lead to a cervical or uterine perforation. This study introduces a novel VR training platform for office hysteroscopy. The presented system was tested in the laboratory setting to prove the feasibility of using VR simulation for office hysteroscopy training. Conducted experiments demonstrated the potential of the system to transfer the essential skills and confirmed the set of proposed metrics for effective assessment.
\end{abstract}

Keywords: Virtual reality · Surgical training $\cdot$ Hysteroscopy $\cdot$ Gynecology $\cdot$ Haptics.

\section{Introduction}

Hysteroscopy is a widely used gynaecological procedure to evaluate and treat cervical and intra-uterine pathology (figure 1). Today, hysteroscopy includes a whole series of diagnostic and operative procedures, including polypectomy, myomectomy, adhesiolysis, treatment of cervical stenosis, treatment of uterine anomalies and many more. Technical refinements, especially in the optics technology and the reduction of the outer diameter of the instrument, have made it possible to 
perform many hysteroscopic procedures, including some operative procedures, in the office setting and without anesthesia. This approach is referred to as office hysteroscopy. Office hysteroscopy is performed with a hysteroscope, which is a long, small-diameter endoscope connected to a light source. A camera is attached to the proximal end of the hysteroscope to transmit the image onto a monitor. The procedure starts in the vagina. In the case of vaginoscopic approach, no speculum is used[1]. The cervix is visualized and the hysteroscope is introduced into the cervical canal without dilatation. Then, under direct visualization, the instrument is advanced step by step into the uterine cavity. Passing the cervical canal without prior dilatation is a thorough procedure requiring a high level of dexterity. In the presence of risk factors, such as anatomical variation, stenotic cervix, postmenopause, previous cesarean section or conisation, passage of the cervical canal can be even more difficult and attention has to be paid to avoid perforation[2].

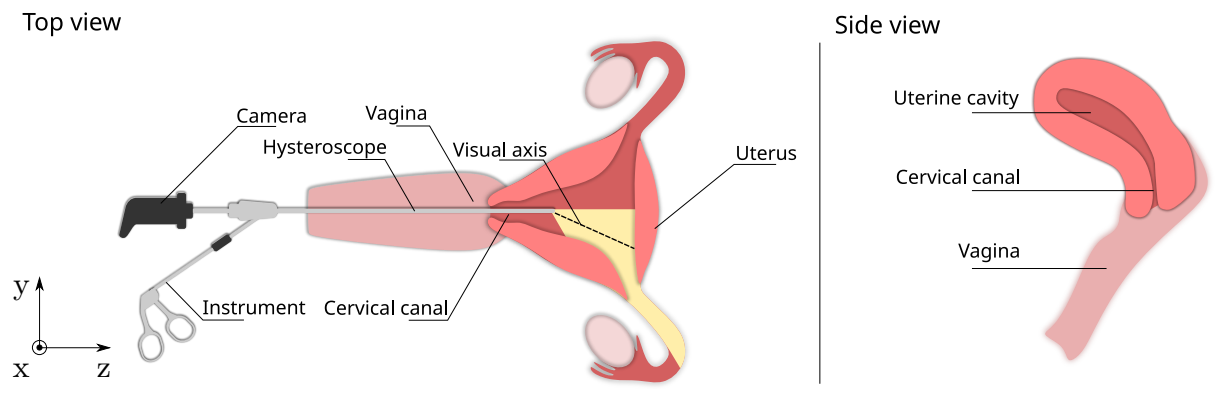

Fig. 1. The schematic diagram of hysteroscopy (top and side views). The hysteroscope is inserted through the vaginal canal and the endocervix to perform treatment in the uterine cavity.

In order to acquire the necessary skills in office hysteroscopy, both theoretical knowledge and practical surgical training are essential. The options for surgical training in hysteroscopy mostly include artificial platforms: physical simulators, also known as box trainers, and virtual reality (VR) simulators. Compared to physical simulators, VR training can potentially provide a higher level of immersion [3]. VR enables rendering of various clinical scenarios in the digital scene with minimal setup time, whereas physical simulators would require considerable workloads to mimic complex pathological conditions. Yet, to the best of our knowledge, no research has been done in VR training for hysteroscopic passage of the endocervix with an office setup. Consequently, surgical treatment performed inside the cervical canal, including biopsy, polyp removal, and surgical treatment of a stenotic cervix, has been left out in the current VR training systems.

The hypothesis of this study is that VR training is capable of transferring the essential skills needed to perform an outpatient hysteroscopic intervention. To prove this hypothesis, the first steps towards the development of a VR surgical 
simulation system designed specifically for office hysteroscopy training are described. The proposed system integrates the real-time physics simulation framework SOFA [4] with Filament [5], a real-time physics-based rendering (PBR) engine. This resulted in a high fidelity VR simulation environment that is close to the actual clinical environment. The developed system was tested and evaluated by experiments in a laboratory setting, which indicates that the proposed training system has a great potential to fill the research gap and to be applied in clinical practice in the mid-term future.

\section{Related works}

A broad body of research has been dedicated to VR surgical training in various domains. Most types of endoscopic procedures share a common set of the basic required skills. Hence, similar assessment methods and metrics can be encountered in such fields as laparoscopy, arthroscopy, ureteroscopy and hysteroscopy training. These metrics typically belong to one of the three following categories: time-based metrics, position-based metrics, and force-based metrics. Time-based metrics are one of the most common metrics. Generally, the total time of performing a certain task or an entire experiment is measured, such as total execution time [6], [7] or phase execution time[8].

Position-based metrics involve a user's performance attributes related to the absolute or relative position of instruments in the simulation. Position-based metrics can indicate task completion rate, for instance, the proximity of the deployed intra-uterine device (IUD) to fallopian tubes ostium in the case of an IUD insertion exercise; or the level of dexterity in motion, as in the simulator presented by Bajka et al. [6] Other studies, such as [9], [10], also employ this type of metric in the training process.

Force-based metrics aim to evaluate a user's performance based on forces that are generated by physical or virtual interaction between instruments and operated tissues. Force-based metrics are part of assessment frameworks presented in [11], [12]. Evaluating force in the simulation imposes additional requirements, namely precise physics modeling methods and realistic haptic feedback. The latter requirement implies that the system should be capable of giving the user kinaesthetic sensations that are close to the real feeling and providing him/her with information about the applied pressure.

Research on VR surgical training for hysteroscopy revolves mainly around works that describe validation experiments on either of two commercial simulators: $\mathrm{GynoS}^{\mathrm{TM}}$ (Virtamed, Switzerland) and HystMentor ${ }^{\mathrm{TM}}$ (3D Systems, U.S.) [8], [13], [14]. The training program of these platforms includes exercises on diagnostic and operative hysteroscopy: polypectomy, myoma resection, septoplasty. Both hysteroscopy training systems focus on surgical training in the OR setup, assuming that prior dilation of the cervix has been performed before the procedure, thus bypassing the endocervix passage phase in the exercises. However, the endocervix passage phase plays an important role in the entire procedure. 
Clinicians require a considerable amount of practice to become proficient in performing this operation and to be able to avoid all potential complications.

Savran et al. presented a study on ensuring the basic hysteroscopic competency using the HystMentor ${ }^{T M}$ simulation system. Participants were given a pass/fail score based on a multimodal assessment system. Reported results showed that false positive and false negative scores were $6.7 \%$ and $27.3 \%$, respectively [13]. Thus, the established metrics still can be improved for more precise assessment. Panel et al. conducted a comparative analysis of overall and subset scores for novice and expert users when performing four types of hysteroscopic intervention in HystMentor ${ }^{\mathrm{TM}}$ : polypectomy, myomectomy, rollerball endometrial ablation and septum resection [8]. The overall multi-metric scores were statistically different between the mentioned groups for three out of four exercises. Panel et al. considered that experienced users obtained low scores for the septum resection exercise due to the incorrect choice of recommended treatment type in the assessment system. Subset scores also demonstrated certain ambiguity. Whilst fluid handling score and economy score demonstrated significant difference between novices and experts, other scores failed to distinguish between the two groups. This ambiguity has also been highlighted by Neis et al. [15] and Elessawy et al. [16], where other subset scores demonstrated no statistical difference.

Apart from objective assessment, another important feature required for effective VR hysteroscopy training is haptic feedback. This particular topic has not been widely addressed in the literature. Bajka et al. presented the HystSim simulation system for diagnostic and operative hysteroscopy [17]. The HystSim platform is augmented with active haptic feedback, allowing its users to perceive forces generated by the interaction between the instrument and objects in the scene. This feature, however, was reported to be in the early stage of development, and thus, according to Bajka et al., might not provide a sufficient level of realism. Moreover, the developed haptic interface allows motions in four degrees of freedoms (DOFs), constraining translation in two directions around the pivot point. However, four DOFs are no sufficient in the case of an office procedure, as the instrument should be capable of unconstrained motion in six DOFs when passing through the cervical canal.

To summarize, there are several gaps in the current methods of VR training for hysteroscopy. First, the training methods for office hysteroscopy fully exclude the passage of the cervical canal and treatment of endocervical pathologies from the training process. Second, presented systems do not provide an active haptic interface suitable for simulating outpatient hysteroscopic interventions.

Based on the mentioned points, in this paper, we aim to design a simulation platform for office hysteroscopy to achieve a high level of haptic and graphical realism. The goal is to design a system that allows clinicians to exercise manipulation of a hysteroscope when passing through the cervical canal and treatment of endocervical and intrauterine pathologies with the office setup. Eventually, the developed VR surgical training system can be used to improve surgical skills in outpatient hysteroscopic intervention. 


\section{Materials and methods}

In this study, a simulation platform was designed, which features a spatial navigation and instrument manipulation task. The presented VR simulation system mimics an office hysteroscopic procedure, starting from locating the external os of the cervix, then passing through the cervical canal and operating inside the uterine cavity. With this exercise, the authors aimed to design an approach for medical students to learn how to safely introduce a hysteroscope inside the uterine cavity and perform simple surgical procedures, such as biopsy or polyp removal inside the cavity and the cervical canal.

Participants should pass through the cervical canal and perform tasks inside the uterine cavity. In this context, several spherical objects located on the surface of the endocervix and inside the uterine cavity were regarded as checkpoints, on which participants had to perform a grasping manipulation.

\subsection{Purpose of the exercise}

The aim of the exercise is to enter the uterine cavity and collect ten checkpoints while maintaining a minimal level of applied force. In the VR training scene, checkpoints are rendered as one millimeter green spheres, which are evenly distributed along the surface of the cervical canal and the uterine cavity. The starting position of the checkpoints is always the same, so the minimal path length does not change. A user can collect checkpoints by grasping them with a hysteroscopic forceps: once a checkpoint is collected, it disappears. When all checkpoints are collected, the exercise is terminated.

\subsection{Hardware setup}

Figure 2 depicts the hardware layout of the system. A user operates the Touch ${ }^{\top M}$ haptic interface (3D Systems Inc., U.S.) to manipulate the hysteroscope in the VR environment in six DOFs and the keyboard to control the forceps in two DOFs, including one translation along the hysteroscope and one joint at the distal part of the forceps to control grasping motion. The Touch ${ }^{T M}$ interface controls the position of the virtual hysteroscope and provides haptic feedback to the user. Up and down arrows on the keyboard are mapped to deploying and retracting motion of the forceps, respectively. Left and right arrows control the grasping motion. A PC (Intel Core I7-9850H, NVIDIA Quadro T1000 w/4GB GDDR6, $8 \mathrm{~GB}$ RAM) runs the simulation and renders $3 \mathrm{D}$ visual representation on a screen from the perspective of a virtual camera attached to the tip of the hysteroscope. The virtual camera is rotated 12 degrees around the $x$ axis of the hysteroscope (figure 1), which corresponds to the typical optical angle of a hysteroscope[18].

\subsection{Scene generation}

The Visible Human cryosection dataset was used to acquire the geometrical models of the uterus [19]. The visual mesh was obtained using manual segmentation in ImageJ [20] and subsequently optimised using quadric collapse edge 


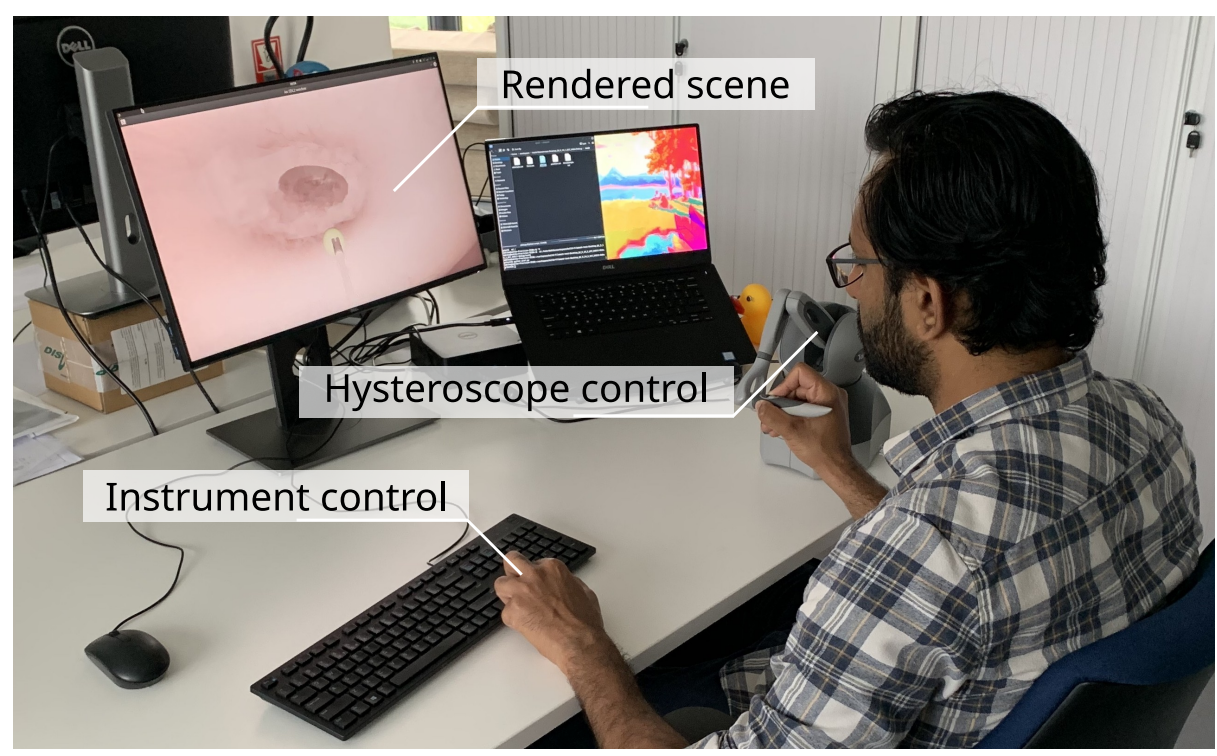

Fig. 2. The illustration of the VR simulation system for office hysteroscopy training.

decimation method in Meshlab [21] in order to reduce the number of vertices, resulting in 22893 elements. Three models depicted in figure 3 were used to simulate different aspects of body behaviour: (1) the visual model, responsible for visual appearance of the uterus; (2) the structural model, used to simulate deformations; and (3) the collision model, detecting intersections with other objects in the simulation. The structural mesh of the uterus was created from the visual mesh using CGAL software [22], which generated an object containing 1310 elements in total. Mechanical deformation of the uterus was modeled in SOFA with the finite element method (FEM) using the structural mesh. In order to optimize the collision pipeline, a simplified version of the visual mesh was used for collision detection containing 900 polygons.

Mechanical properties of the uterus were obtained from quasi in-vivo measurements performed by Omari et al. [23]. The Young's modulus of the organ was set to $12 \mathrm{kPa}$. The mass was assumed to be uniformly distributed between the structural elements in the body. To preserve the position of the body in the scene, the uterus is rigidly fixed in space at left and right ostia.

The hysteroscope is simulated as a cylinder, four millimeters in diameter and 130 millimeters in length, with a virtual camera attached to the tip and angled at 12 degrees. In order to improve stability of the simulation and haptic feedback, the haptic interface controls the position of the hysteroscope via the proxy model. The proxy model does not have a collision or a visual representation in the simulation, but rather copies the position of the haptic interface and attracts the hysteroscope to it via a generic six DOF spring. This method ensures that no discrete change of the position occurs in the simulation. The forceps are 


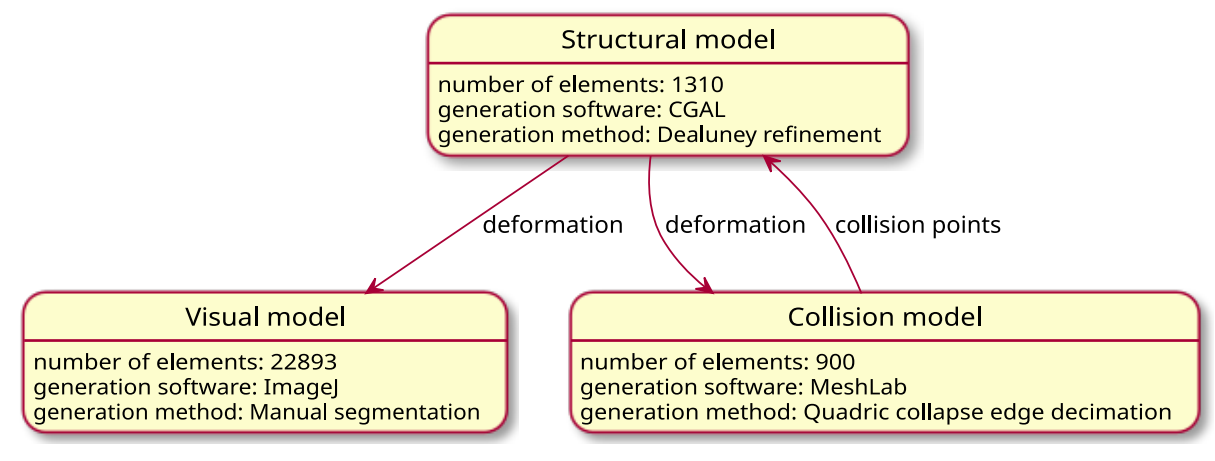

Fig. 3. The multi-model representation diagram. Links denote the relations between the models. Nodes specify the number of elements in the model, the generation software and the generation method.

simulated as an articulated rigid body, one millimeter in diameter, with two DOF and the base attached to the hysteroscope.

Checkpoints were represented as one-millimeter green spheres. The total number of the checkpoints was ten, with five checkpoints located in the cervical canal and five checkpoints located in the uterine cavity. The initial position of all checkpoints was the same for each experiment. However, as the geometry of the uterus can change due to tissue deformation, checkpoint positions should be also updated with each simulation step. To solve this problem, each checkpoint is mapped to a subset of uterus vertices around it, thus preserving the relative position with respect to the uterus during the simulation. The barycentric mapping was used to update the checkpoints position [24].

\subsection{Software architecture}

The software architecture of the simulator consists of three main components: the physics engine, the visual renderer and the haptic loop. All mentioned components run in separate threads as all of them have different timing requirements.

The SOFA framework [4] is used as the physics engine of the system. It runs at the update rate of approximately 150 frames per second (FPS) and performs collision detection, structural deformation simulation, and mapping between models. The mechanical model is represented by a tetrahedral mesh, which is mapped to the collision model and the visual model polygon meshes. The geometry of the models is synchronised using the barycentric mapping.

The Filament renderer [5] is used for 3D graphical representation. Figure 4 depicts an example of a rendered scene in the simulation. The visual server runs at 50 FPS and is fully decoupled from the physics simulation. The physics engine passes the geometry of each object to the visual server at the initialisation phase. During simulation, the visual server queries updates of the objects geometry at the start of each iteration using a mediator class, which ensures decoupling between these components. 


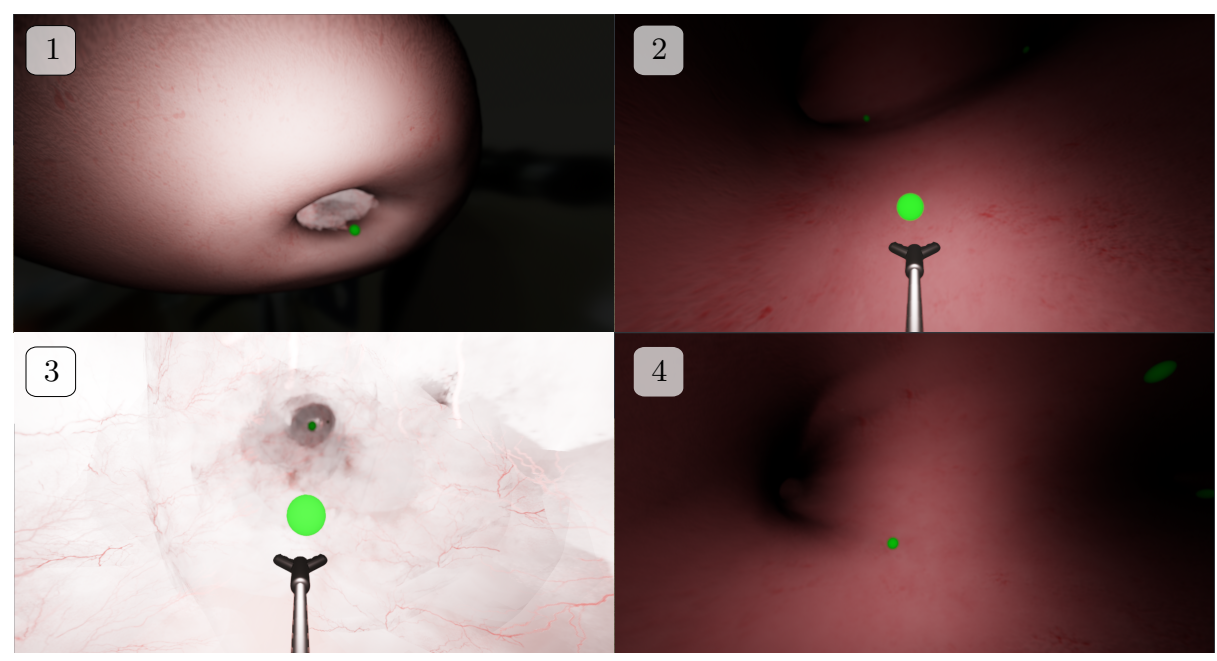

Fig. 4. The examples of the rendered scene: (1) external os, (2) uterine cavity, (3) cervical canal, (4) fundus and left ostium.

Finally, the haptic loop is implemented using the OpenHaptics [25] libraries. In order to provide realistic haptic feedback, the haptic loop should run at the update rate of $1 \mathrm{kHz}$, meaning that it should be detached from the physics simulation. The physics loop is connected to the haptic loop using the LCPForceFeedback component, which utilizes constraints generated by the contact points between the instrument and the uterus to calculate force feedback at the required update rate.

\subsection{Experiments and assessment metrics}

Ten subjects, eight male and two female, with no prior experience in hysteroscopy participated in the study to evaluate the proposed concept. The subjects had no to moderate gaming and virtual reality experience. Assessment of a subject's performance was based on following metrics: total number of grasping attempts $\left(n_{a}\right)$, total execution time $\left(t_{e}\right)$, cervical canal passage time $\left(t_{c}\right)$, total trajectory length $\left(l_{t}\right)$, effective jerk (mean jerk excluding idle states) $\left(j_{e}\right)$, effective force (mean force excluding idle states) $\left(f_{e}\right)$, cumulative force (integrated force value) $\left(f_{c}\right)$, and force fast Fourier transform $(\mathrm{FFT})\left(f_{F F T}\right)$. The latter metric is the cumulative sum of the real component of applied force in the frequency domain.

Each participant performed five repetitive trials of the exercise to investigate the potential of transferring the essential skills for office hysteroscopy in the proposed system. Each participant was asked to take the required time to rest between trials. To check statistical significance, the obtained results were tested using the Mann-Whitney U-test. A metric was considered to be significant when the significance level was less than or equal to 0.05 . 


\section{Results}

Table 1 shows the measured metrics across all participants for each trial. Mean and standard deviation were calculated for each metric. The $p$-values that were calculated between the first and the last trial are listed in the last column and denoted with an asterisk symbol $(*)$ if a metric has statistical significance $(p \leq 0.05)$. Among nine metrics, five demonstrated statistical significance: total execution time $t_{e}$, cervical canal passage time $t_{c}$, total trajectory length $l_{t}$, cumulative force $f_{c}$, and total number of grasping attempts $n_{a}$. Force FFT $f_{F F T}$ also showed a high level of significance, although the p-value did not reach the required level. These metrics are depicted in figure 5.

Table 1. Mean and standard deviation of the experiments across all participants for each trial. The $p$-values were calculated between the first and the last trial.

\begin{tabular}{l|c|c|c|c|c|c}
\multirow{2}{*}{ Metric } & \multicolumn{5}{|c|}{ Trial number } & $p$-value \\
\cline { 2 - 6 } & 1 & 2 & 3 & 4 & 5 & \\
\hline$n_{a}$ & $44.67 \pm 10.37$ & $42.44 \pm 18.37$ & $37.11 \pm 7.49$ & $32.44 \pm 5.72$ & $36.00 \pm 9.93$ & $0.037 *$ \\
$t_{e}[s]$ & $248.7 \pm 78.9$ & $205.1 \pm 62.7$ & $179.9 \pm 60.9$ & $155.5 \pm 37.7$ & $143.2 \pm 46.6$ & $0.002 *$ \\
$t_{c}[\mathrm{~s}]$ & $168.2 \pm 76.6$ & $119.3 \pm 35.0$ & $105.0 \pm 43.8$ & $93.7 \pm 30.5$ & $88.1 \pm 43.1$ & $0.006 *$ \\
$l_{t}[\mathrm{~cm}]$ & $41.36 \pm 22.32$ & $36.34 \pm 16.65$ & $34.55 \pm 15.75$ & $34.37 \pm 23.31$ & $33.43 \pm 28.99$ & $0.046 *$ \\
$j_{e}\left[\mathrm{~km} / \mathrm{s}^{3}\right]$ & $10.76 \pm 0.81$ & $11.09 \pm 0.77$ & $11.36 \pm 1.35$ & $12.24 \pm 3.21$ & $11.94 \pm 1.92$ & 0.961 \\
$f_{e}[N]$ & $0.62 \pm 0.08$ & $0.62 \pm 0.06$ & $0.65 \pm 0.09$ & $0.63 \pm 0.09$ & $0.67 \pm 0.11$ & 0.760 \\
$f_{c}[N \cdot s]$ & $133.1 \pm 48.4$ & $108.2 \pm 32.8$ & $105.6 \pm 38.8$ & $85.2 \pm 31.0$ & $87.2 \pm 52.8$ & $0.021 *$ \\
$f_{F F T}$ & $10.18 \pm 3.40$ & $9.49 \pm 2.58$ & $9.26 \pm 2.79$ & $9.02 \pm 2.78$ & $8.85 \pm 3.99$ & 0.092
\end{tabular}

Three participants reported to experience fatigue during the experiments, which was confirmed by the recorded drop in performance. However, the causeeffect relationship between fatigue and performance is out of the scope of the presented study.

\section{Discussion}

Overall, the proposed system demonstrated skill acquisition capabilities. Timebased metrics (total execution time and cervical canal passage time) showed the biggest change, gradually decreasing both in mean and standard deviation. These findings correlate with previous studies in other domains of hysterscopic 

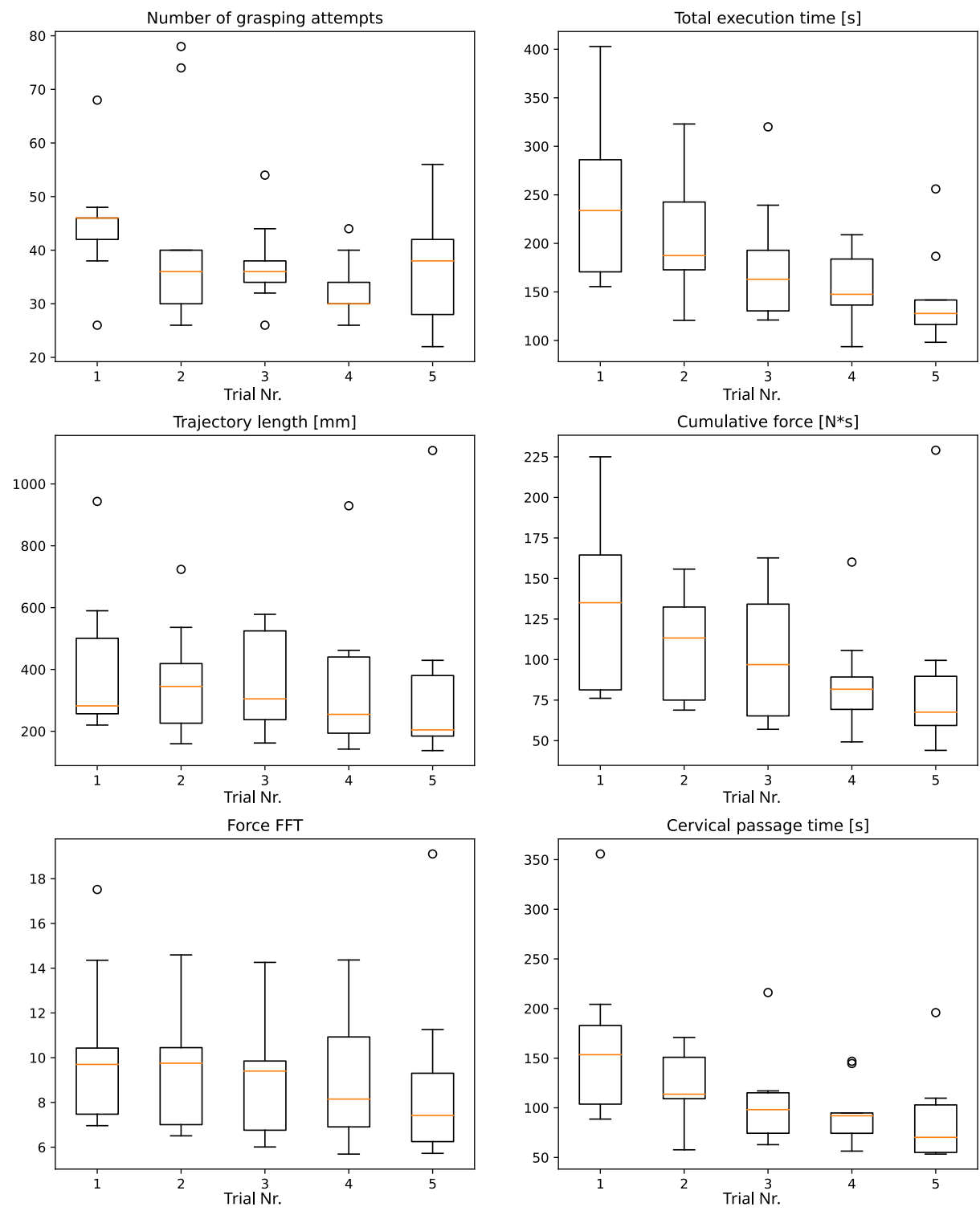

Fig. 5. Box plots showing minimum, maximum, median, and interquartile range values of the statistically significant metrics for each trial.

training, in which total procedure time demonstrated significant difference between novices and expert clinicians [8]. The results also indicated another important metric to effectively assess a subject's performance: cumulative force. Both mean and standard deviation of this metric significantly improved with each trial, although the progress was somewhat slower compared to pure time-based 
metrics (figure 5). Being both a time- and a force-based metric, this value can better estimate the level of patient comfort and thus become an effective tool for cross-correlation assessment in the overall score.

Likewise, trajectory length and number of attempts exhibited gradual improvement over trials. However, the slope of the progress was not as smooth as in the case of time-based metrics (figure 5). The mean trajectory length of the second and third trials was higher compared to the first trial, whereas standard deviation and interquartile range also increased in values. This effect might be partially related to growing tiredness, as the drop of performance in these parameters was most observable in participants that reported to experience fatigue.

Opposed to the initial assumption of providing insights about the same phenomenon, tremor, force FFT and effective jerk behaved in a different manner. Whereas force FFT improved with each trial, effective jerk remained on the same level, although individual for each participant.

Contrarily to previous studies in VR hysteroscopic simulation with the same number of trials and non-expert participants [6], [8], a plateau effect was not observed for the most number of statistically significant metrics. Mean and standard deviation of statistically significant metrics decreased with each trial without a significant change of slope. We can assume that this effect should take place with a higher number of repetitions, but additional research is required.

No significant change was observed in effective force over repetitions. This type of behaviour was somehow expected, as the exercise was designed for novice users and did not include the cases of a stenotic cervix. The authors assume that the situation might drastically change after augmenting the haptic interface with a mock-up of a hysteroscope. Due to introduction of a pivot point, motion in two rotational DOFs will be inverted and force perception will be partially reduced due to the lever effect.

\section{Conclusion and Future Work}

This paper presents some first results on the feasibility of VR training for office hysteroscopy. The developed platform aims to introduce an effective approach allowing medical students to sharpen their skills in performing outpatient hysteroscopic treatment. Employing physics-based rendering techniques along with realistic haptic feedback contributes to the level of realism and significantly improves the transfer of training. The preliminary results demonstrate the ability of the simulator to develop a trainee's dexterity in passage of the cervical canal and manipulation of hysteroscopic instruments. However, some of the presented metrics need further refinement.

Future work will focus on the face and content validity of the system. The hardware setup should be updated by adding a phantom of the female reproductive system, serving as the fulcrum point and contributing to visual resemblance between the simulation and the procedure. The haptic interface should be augmented with a mock-up of a real hysteroscopic instrumentation setup for higher content validity. 
From the software perspective, the simulation also requires particular improvements. A graphical interface can enhance the usability of the system, making it easier to interact for a non-technical user. Another important consideration is to add variability by introducing new exercises and changing the simulation parameters in accordance with the level of complexity. Finally, the assessment system can be refined into a framework for effective overall evaluation and learning curve estimation. Introducing a complete solution for automatic assessment will serve as the foundation for adaptive training program, when the level of complexity grows over time with the level of a trainee's competence.

Overall, the proposed system has demonstrated its feasibility in VR surgical training for office hysteroscopy. Future studies will focus on further design and validation of the system with medical staff. These improvements will contribute to creating a useful surgical training system with a high level of realism and efficient transfer of skills characteristics.

\section{References}

[1] S. Bettocchi and L. Selvaggi, "A vaginoscopic approach to reduce the pain of office hysteroscopy," Journal of the American Association of Gynecologic Laparoscopists, vol. 4, no. 2, pp. 255-258, Feb. 1997, ISSN: 10743804. DOI: 10.1016/S1074-3804(97)80019-9.

[2] M. G. Munro and L. A. Christianson, "Complications of hysteroscopic and uterine resectoscopic surgery," Clinical Obstetrics and Gynecology, vol. 58, no. 4, pp. 765-797, 2015, ISSN: 15325520. DOI: 10 . 1097 / GRF . 0000000000000146.

[3] M. M. Erian, G. R. McLaren, and A. M. Erian, "Advanced hysteroscopic surgery training," Journal of the Society of Laparoendoscopic Surgeons, vol. 18, no. 4, 2015, ISSN: 19383797. DOI: 10.4293/JSLS. 2014.00396.

[4] F. Faure, C. Duriez, H. Delingette, J. Allard, B. Gilles, S. Marchesseau, H. Talbot, H. Courtecuisse, G. Bousquet, I. Peterlik, and S. Cotin, "SOFA: A Multi-Model Framework for Interactive Physical Simulation," in, 2012, pp. 283-321. DOI: 10.1007/8415_2012_125.

[5] Google, Filament rendering engine, Accessed: 2020-05-10, 2020. [Online]. Available: https://github.com/google/filament.

[6] M. Bajka, S. Tuchschmid, D. Fink, G. Székely, and M. Harders, "Establishing construct validity of a virtual-reality training simulator for hysteroscopy via a multimetric scoring system," Surgical Endoscopy, vol. 24, no. 1, pp. 79-88, 2010, ISSN: 14322218. DOI: 10.1007/s00464-009-0582-4.

[7] A. Hackethal, M. Immenroth, and T. Bu“rgerbu"rger, "Evaluation of target scores and benchmarks for the traversal task scenario of the minimally invasive surgical trainer-virtual reality (MIST-VR) laparoscopy simulator," DOI: 10.1007/s00464-004-2224-1.

[8] P. Panel, M. E. Neveu, C. Villain, F. Debras, H. Fernandez, and E. Debras, "Hysteroscopic resection on virtual reality simulator: What do we measure?" Journal of Gynecology Obstetrics and Human Reproduction, 
vol. 47, no. 6, pp. 247-252, Jun. 2018, ISSN: 24687847. DOI: 10 . 1016 / j.jogoh.2018.02.005.

[9] T. Anderson, M. Thomassee, A. Karhade, J. Peacock, M. Tolish, and F. Young, "Development of a Low Cost, Medium Fidelity Hysteroscopy Simulator for Spatial Orientation Training: Proof of Concept," Journal of Minimally Invasive Gynecology, vol. 22, no. 6, S43, Nov. 2015, ISSN: 15534650. DOI: $10.1016 / j \cdot j m i g .2015 .08 .118$.

[10] S. Bayona, J. M. Fernández-Arroyo, I. Martín, and P. Bayona, "Assessment study of insight ARTHRO VRR arthroscopy virtual training simulator: Face, content, and construct validities," Journal of Robotic Surgery, vol. 2, no. 3, pp. 151-158, Sep. 2008, ISSN: 18632483. DOI: $10.1007 /$ s11701-0080101-y.

[11] R. B. Singapogu, S. Dubose, L. O. Long, D. E. Smith, T. C. Burg, C. C. Pagano, and K. J. Burg, "Salient haptic skills trainer: Initial validation of a novel simulator for training force-based laparoscopic surgical skills," Surgical Endoscopy, vol. 27, no. 5, pp. 1653-1661, 2013, ISSN: 14322218. DOI: $10.1007 / \mathrm{s} 00464-012-2648-y$.

[12] T. Horeman, S. P. Rodrigues, F. Willem Jansen, J. Dankelman, and J. J. Van Den Dobbelsteen, "Force parameters for skills assessment in laparoscopy," IEEE Transactions on Haptics, vol. 5, no. 4, pp. 312-322, 2012, ISSN: 19391412. DOI: 10.1109/TOH.2011.60.

[13] M. M. Savran, A. B. Nielsen, B. B. Poulsen, P. B. Thorsen, and L. Konge, "Using virtual-reality simulation to ensure basic competence in hysteroscopy," Surgical Endoscopy, vol. 33, no. 7, pp. 2162-2168, Jul. 2019, ISSN: 14322218. DOI: 10 . 1007/s00464-018-6495-3. [Online]. Available: https : //doi . org/10.1007/s00464-018-6495-3.

[14] M. G. Munro and D. P. Behling, "Virtual reality uterine resectoscopic simulator: Face and construct validation and comparative evaluation in an educational environment," Journal of the Society of Laparoendoscopic Surgeons, vol. 15, no. 2, pp. 142-146, Apr. 2011, ISSN: 10868089. DOI: 10. 4293/108680811X13071180406312. [Online]. Available: /pmc/articles/ PMC3148859/?report=abstract\%20https : / / www . ncbi . nlm . nih . gov / pmc/articles/PMC3148859/.

[15] F. Neis, S. Brucker, M. Henes, •. F. A. Taran, S. Hoffmann, M. Wallwiener, •. B. Schönfisch, N. Ziegler, A. Larbig, •. Rudy, and L. De Wilde, "Evaluation of the HystSim TM-virtual reality trainer: an essential additional tool to train hysteroscopic skills outside the operation theater," DOI: 10.1007/s00464-016-4837-6.

[16] M. Elessawy, M. Skrzipczyk, C. Eckmann-Scholz, N. Maass, L. Mettler, V. Guenther, M. van Mackelenbergh, D. O. Bauerschlag, and I. Alkatout, "Integration and Validation of Hysteroscopy Simulation in the Surgical Training Curriculum," Journal of Surgical Education, vol. 74, no. 1, pp. 8490, Jan. 2017, ISSN: 18787452. DOI: 10.1016/j.jsurg.2016.06.007.

[17] M. Bajka, S. Tuchschmid, M. Streich, D. Fink, G. Szèkely, and M. Harders, "Evaluation of a new virtual-reality training simulator for hysteroscopy," 
Surgical Endoscopy, vol. 23, no. 9, pp. 2026-2033, 2009, ISSN: 14322218. DOI: $10.1007 / \mathrm{s} 00464-008-9927-7$.

[18] A. U. Hernandez, "In-office hysteroscopy," in Hysteroscopy, Springer International Publishing, Feb. 2018, pp. 33-40, ISBN: 9783319575599. DOI: 10.1007/978-3-319-57559-9_4. [Online]. Available: https://doi.org/ $10.1007 / 978-3-319-57559-9 \% 7 \mathrm{~B} \% 5 C_{-} \% 7 \mathrm{D} 4$.

[19] M. J. Ackerman, "The visible human project," Proceedings of the IEEE, vol. 86, no. 3, pp. 504-511, 1998, ISSN: 00189219. DOI: 10.1109/5.662875.

[20] C. A. Schneider, W. S. Rasband, and K. W. Eliceiri, NIH Image to ImageJ: 25 years of image analysis, Jul. 2012. DOI: 10.1038/nmeth. 2089. [Online]. Available: https://www. nature.com/articles/nmeth. 2089.

[21] P. Cignoni, M. Callieri, M. Corsini, M. Dellepiane, F. Ganovelli, and G. Ranzuglia, "MeshLab: an Open-Source Mesh Processing Tool," in Eurographics Italian Chapter Conference, V. Scarano, R. D. Chiara, and U. Erra, Eds., The Eurographics Association, 2008, ISBN: 978-3-905673-68-5. DOI: 10 . 2312/LocalChapterEvents/ItalChap/ItalianChapConf2008/ $129-136$.

[22] P. Alliez and A. Fabri, "CGAL-The computational geometry algorithms library," in ACM SIGGRAPH 2016 Courses, SIGGRAPH 2016, Association for Computing Machinery, Inc, Jul. 2016, ISBN: 9781450342896. DOI: $10.1145 / 2897826.2927362$.

[23] E. A. Omari, T. Varghese, M. A. Kliewer, J. Harter, and E. M. Hartenbach, "Dynamic and quasi-static mechanical testing for characterization of the viscoelastic properties of human uterine tissue," Journal of Biomechanics, vol. 48, no. 10, pp. 1730-1736, Jul. 2015, ISSN: 0021-9290. DOI: 10. 1016/J . JBIOMECH . 2015.05. 013. [Online]. Available: https : //www . sciencedirect.com/science/article/pii/S0021929015002900.

[24] M. S. Floater and J. Kosinka, "Barycentric interpolation and mappings on smooth convex domains," in Proceedings - 14th ACM Symposium on Solid and Physical Modeling, SPM'10, New York, New York, USA: ACM Press, 2010, pp. 111-116, ISBN: 9781605589848. DOI: 10.1145/1839778.1839794. [Online]. Available: http : / / portal . acm . org / citation . cfm ? doid= 1839778.1839794.

[25] 3DSystems, Geomagic openhatpics, Accessed: 2020-05-10, 2020. [Online]. Available: https://www.3dsystems.com/haptics-devices/openhaptics. 